\title{
The Effectiveness Mandala Therapy On Coping Stress
}

\author{
Hasnida1, Amalia Meutia ${ }^{2}$ \\ Facultyof Psychology, Universitas Sumatera Utara \\ Jl. Dr. Mansyur No.7, \\ Medan - Indonesia \\ hasnida_hasan@yahoo.com
}

\begin{abstract}
Mandala as therapeutic intervention commonly used by ordinary people and therapists to reduce psychological stress. However, in Indonesia particularly in Medan, there is no scientificalevidence-based on empirical research of itis in treatment related to coping stress. This wasan explanatory study that examined the effectiveness of Mandala coloring therapy in thereduction of stress level. The stress level was measured by the Perceived Stress Scale at baseline and after coloring. A sample of 32 adult participants assigned to coloring a Mandala pattern in three times experimental condition. Results findings suggest that structured coloring of Mandala pattern induce a relaxed state that decreased stress level.
\end{abstract}

Keyword : stress, coping stress, coloring therapy

\section{INTRODUCTION}

Psychological stress refers to a relationship with the environment that the person appraises as significant for his or her well-being and in which the demands tax or exceed available coping resources (Lazarus and Folkman,1986). Lazarus defines coping as the cognitive and behavioral efforts made to master, tolerate, or reduce external and internal demands and conflicts among them. They can attempt to change the person-environmentrealities of negative emotions or stress (problem-focused coping). They can also relate to internal elements and try to reduce a negative emotional state or change the appraisal of the demanding situation (emotion-focused coping). (Lazarus and Folkman, 1984).Relaxation as part of emotion-focused coping can be done by meditation, music therapy, respiratory therapy, laughter therapy, worship therapy and art therapy (Hallowell, 2007).

Recently, a new trend emerged in coping stress, namely the circulation of coloring books for adults which claimed by the author as a form of art therapy that could be one alternative coping stress. The book is devoted to adults and is different from coloring books for children. Even the drawings are drawings that have a high level of complexity. However, the effectiveness of these coloring books in reducing stress is still not much scientifically proven, especially in Indonesia.
The research that has been conducted on this trend has been developed by Rodski (2012) using coloring therapy whose concept is taken from art therapy as a medium for relaxation and meditation. The results suggested that there was an increase in stress management in groups that received coloring therapy treatments. Research on color therapy in adults is also investigated by Flettet al. (2017) who conclude that daily coloring can improve some negative psychological outcomes and that it may provide an effective, inexpensive, and highly accessible self-help tool for nonclinical samples. The same results were also obtained from Eaten \&Tieber research (2017) which showed positive effects of coloring with greater anxiety reduction. It suggests that well-being might be facilitated by a coloring task that balances structure and engagement.

This research will use Mandala pattern. Mandala is from Hindu word derived from Sanskrit meaning circle or center. Jung believed mandala to be an archetype of the "Self," which he defined as an organizing center where psychic growth comes from. This inner center was a guiding factor different from the conscious personality that enabled a person to become a complete human being (Slegelis, 1987). The mandala has been widely accepted as an effective art therapy tool for the diagnosis and treatment of emotional or psychological status, symptoms, and disorders. There have also been many studies showing that a mandala is an effective tool for therapeutic purposes. (Kimet al., 2009). Research 
conducted by Curry \& Kasser (2011) showed that structured coloring of Mandala pattern might induce a meditative state that benefits individuals are suffering from anxiety. The same thing was also stated Babouchkina \& Robbins (2015) who conducted experimental research which the results demonstrated that the circular shape of the mandala serves as an "active ingredient" in mood enhancement.

\section{METHODS AND PARTICIPANTS}

Participants were 32 people aged between 21-40 years old, consisted of 21 women and 11 men. Participants were divided into two groups, 21 of whom were lecturers, and the remaining 11 were college students.

\section{PROCEDURE}

The number of participants before the pretest were 58 people. Participants are divided into 2 groups, namely groups of lecturers and college students. After completing consent procedures, the pretest was performed to the participants, namely completing the 10 items of the Perceived Stress Scale, which was adapted from Cohenet al. (1988) measure. Respondents rated their current level of stress on a 5-point scale, ranging from $0=$ Never, $1=$ Almost Never, 2 = Sometimes, 3 = Fairly Often, $4=$ Very Often Participants whose stress scores were in the medium and high category then received treatment in the form of Mandala coloring therapy. Only 32 people in this research that were included in the sample category.

Before participants did coloring activities, researchers provided breathing relaxation exercises and visualization techniques aimed at making participants feel more relaxed and comfortable. Subsequently, to each participant, a coloring book was distributed, and they were instructed to select the Mandala pattern they liked best to be colored. They should color the pattern using 24 color pencils. There was no time limit given to participants.

This treatment is done 3 times in 3 meetings. After thethird treatment, all participants once again completed the Perceived Stress Scale as aposttestto assess that any changes in stress that occurred after coloring.

\section{RESULTS}

The ages of experimental group participants varied from 21 to 40 years with an average age of 32 years. The respondent's stress level before being given the Mandala Coloring
Therapy were obtained as follows: 1 person (3\%) was in the category of moderate stress levels, 21 people $(66 \%)$ were in the category of severe stress level, and 10 people (31\%) were in the category of very severe stress levels. Based on these data, it can be seen that the general category of stress is dominated at the level of severe stress.

The respondent's stress level after being given the Mandala Coloring Therapy were obtained as follows: 2 people $(6 \%)$ were in the category of very light stress level, 3 people (9\%) were in the category of mild stress level, 10 people $(31 \%)$ were in the category of moderate stress level, 15 people $(46 \%)$ were in the category of severe stress level and 2 people (6\%) were in the category of very severe stress level. From these data, it can be seen the spread of stress levels in the research respondents after being given the Mandala Coloring Therapy.

Based on paired t-test, the Mean value of respondent's stress level before treatment was 19.41 and the Mean value after treatment was 15.31. If the correlation was viewed, the contribution of Mandala coloring therapy to decrease stress level was $0.380^{2}=0.15(15 \%)$. It indicated that Mandala coloring therapy contributes $15 \%$ to the respondents' stress reduction, while another $85 \%$ was caused by other factors. With probability value or sig.0.00 $<0.05$, it can be concluded that there was a very significant difference in stress levels between before and after being given the Mandala Coloring Therapy in this research respondents.

\section{CONCLUSIONS}

1. Mandala Coloring Therapy managed to reduce the stress level of this research respondents.

2. There was a correlation between stress level of research respondents before being treated with Mandala Coloring Therapy and after being treated with Mandala Coloring Therapy. Correlation contribution to the decrease of stress level was $15 \%$, while $85 \%$ was caused by other factors.

3. Mandala coloring therapy very significantly reduces stress levels in this research respondents.

\section{Suggestion:}

For researchers interested in coloring therapy, to determine the effectiveness of Mandala coloring therapy, further research can be done with various respondents such 
as employees, patients, or housewives, to know the effectiveness of these therapies in different communities.

\section{REFERENCE:}

Anastasia Babouchkina, Steven J. Robbins. (2015). Reducing Negative Mood Through Mandala Creation: A Randomized Controlled Trial. Art Therapy 32:1, pages 34-39.

Cohen, S. and Williamson, G. (1988). Perceived Stress in a Probability Sample of the United States. In Spacapan, S. and Oskamp, S. (Eds.) The Social Psychology of Health. Newbury Park, CA: Sage.

Flett, Jayde A. M., Celia Lie, Benjamin C Riordan, Laura M Thompson, Tamlin S Conner, Harlene Hayne. (2017) Sharpen Your Pencils: Preliminary Evidence that Adult Coloring Reduces Depressive Symptoms and Anxiety. Creativity Research Journal 29:4, pages 409-416.

Hallowell, L. (2007). Art Therapy ProgramChildren Cancer Centre. Retrieved from URL:

http:///www.rch.org.au/ept/art/index.cfm ?doc_id= 7693
Judy Eaton, Christine Tieber. (2017) The Effects of Coloring on Anxiety, Mood, and Perseverance. Art Therapy 34:1, pages 4246.

Kim, Hyung-Seok ., Kim, Youn-Hee (2009). A computer system for art therapy assessment of elements in structured mandala. The Arts in Psychotherapy 36. 19-28

Lazarus, R S and Folkman, S, (1984). Stress, Appraisal, and Coping. New York: Springer.

Lazarus, R S and Folkman, S, (1986). Cognitive theories of stress and the issue of circularity. In M H Appley and R Trumbull (Eds), (1986). Dynamics of Stress. Physiological, Psychological, and Social Perspectives (pp. 63-80). New York, Plenum.

Nancy A. Curry BA \& Tim Kasser Ph.D. Can Coloring Mandala reduce anxiety? Art Therapy Vol. 22 , Iss. 2,2005

Slegelis, M.H. (1987). A Study Of Jung's Mandala And Relationship To Art Psychotherapy. The Arts in Psychotherapy, 14, 103-311.

Rodski, Stan. (2012). Anti Stress, Colouring Book for Adults. http://www.colourtation.com/brain science 